\title{
An atmospheric neutrino flux calculation constrained by measurements of cosmic muon fluxes
}

\author{
Juan Pablo Yáñez ${ }^{a, *}$ and Anatoli Fedynitch ${ }^{b}$ \\ ${ }^{a}$ Dept. of Physics, University of Alberta \\ Edmonton, Alberta, Canada T6G 2E1 \\ ${ }^{b}$ Institute for Cosmic Ray Research, The University of Tokyo, \\ 5-1-5 Kashiwa-no-ha, 277-8582 Kashiwa, Chiba, Japan \\ E-mail: j.p.yanez@ualberta.ca, afedyni@icrr.u-tokyo.ac.jp
}

\begin{abstract}
Atmospheric neutrinos, produced by cosmic ray interactions, are a unique probe to study neutrino oscillations and exotic phenomena beyond the Standard Model, as well as the main background to measurements of astrophysical neutrinos. Due to the higher precision of next generation detectors, flux uncertainties are increasingly impacting such studies, in particular driven by the lack of measurements from hadronic particle production in the very forward region. In this work we introduce constraints provided by inclusive muon flux measurements into the calculations of lepton fluxes provided by the MCEQ code to obtain a new atmospheric neutrino flux prediction.
\end{abstract}

$37^{\text {th }}$ International Cosmic Ray Conference (ICRC 2021)

July 12th-23rd, 2021

Online - Berlin, Germany

\footnotetext{
*Presenter
} 


\section{Introduction}

Atmospheric neutrinos are both a tool for discovery, as demonstrated by their role in uncovering neutrino oscillations and searches for new physics, as well as the main background for the emerging field of neutrino astronomy. Their understanding is central to the interpretation of data from operational neutrino observatories like Super-Kamiokande, IceCube and future projects such as Hyper-Kamiokande, Km3NeT, IceCube-Gen2, GVD and P-ONE, to mention a few. However, despite their relevance, existing calculations from neutrino fluxes have significant uncertainties that are already impacting physics results.

One major sources of uncertainty for neutrino fluxes comes from the limited knowledge of the primary cosmic ray spectrum [1]. Historically the primary cosmic ray flux has been modeled as a collection of multiple nuclei that follow a power-law spectrum, fitting the scaling factor and spectral index to data from multiple experiments that was not always in agreement [2]. The other challenge resides on the phase space of hadronic interactions that is relevant for modeling the flux of atmospheric neutrinos: light meson production at very small scattering angles [3]. This region is the domain of non-perturbative QCD, which means that there are no robust methods to compute them from first principles. Phenomenological models are used instead, but they need to be tuned to data which can only come from fixed target experiments.

One way to reduce these uncertainties is to make use of the correlation between the flux of atmospheric neutrinos and that of cosmic muons, which originate in the same decay of charged mesons, as done first by Honda et al. [4]. In this work we follow a similar method, but we extend it to include flux and muon charge ratio measurements from multiple experiments as calibration data to modify the output of fluxes computed with purely data driven inputs, and thus compute a calibrated atmospheric neutrino flux.

\section{Muon spectrometer data}

The muon data we use were collected by spectrometers as muon fluxes, either inclusive or separated by charge, as well as the ratio $\mu^{+} / \mu^{-}$, both as a function of energy or momentum. Data can be reported for various zenith angles or turned into an equivalent vertical flux, and, for experiments deep underground, are typically transformed to surface fluxes. Since experiments apply corrections to their measurements to report them in this manner, we restrict ourselves to use only data sets with a detailed description of the systematic uncertainties introduced. We furthermore impose an additional constrain on the muon momentum to be higher than $5 \mathrm{GeV}$ at the detector to avoid complications introduced by geomagnetic effects. The initial list of experiments considered for this study are listed in Table 1.

Each of the data sets in Table 1 were studied prior to including them as calibration data. The reported systematic uncertainties were considered either as uncorrelated errors on their measurements, summed in quadrature to the statistical ones, or parametrized as an effect that could be corrected when comparing the data to the calculation. The parametrization of the uncertainties would have been the desired procedure for all experiments, but only the L3+cosmic publications $[8,12]$ contained enough information to do this in full. For the rest of the experiments, a mix of both approaches was used. Additionally, a parameter to correct for potential biases in the energy 


\begin{tabular}{lllllll}
\hline Experiment & Energy $(\mathrm{GeV})$ & Measurements & Reported unit & Location & Altitude & Zenith range \\
\hline AMS-02 [5] & $0.1-2500$ & Flux \& charge ratio & rigidity & $28.57^{\circ} \mathrm{N}, 80.65^{\circ} \mathrm{W}$ & $5 \mathrm{~m}$ (sea level) & unfolded \\
BESS-TeV [6] & $0.6-400$ & Flux & momentum & $36.2^{\circ} \mathrm{N}, 140.1^{\circ} \mathrm{W}$ & $30 \mathrm{~m}$ & $0-25.8^{\circ}$ \\
CMS [7] & $5-1000$ & Charge ratio & momentum & $46.31^{\circ} \mathrm{N}, 6.071^{\circ} \mathrm{E}$ & $420 \mathrm{~m}$ & $p \cos \theta_{z}$ \\
L3+C [8] & $20-3000$ & Flux \& charge ratio & momentum & $46.25^{\circ} \mathrm{N}, 6.02^{\circ} \mathrm{E}$ & $450 \mathrm{~m}$ & $0-58^{\circ}$ \\
DEIS [9] & $5-10000$ & Flux & momentum & $32.11^{\circ} \mathrm{N}, 34.80^{\circ} \mathrm{E}$, & $5 \mathrm{~m}$ (sea level) & $78.1-90^{\circ}$ \\
MINOS [10] & $1000-7000$ & Charge ratio & total energy & $47.82^{\circ} \mathrm{N}, 92.24^{\circ} \mathrm{W}$ & $5 \mathrm{~m}$ (sea level) & unfolded \\
OPERA [11] & $891-7079$ & Charge ratio & total energy & $42.42^{\circ} \mathrm{N}, 13.51^{\circ} \mathrm{E}$ & $5 \mathrm{~m}$ (sea level) & $E \cos \theta^{*}$ \\
\hline
\end{tabular}

Table 1: List of measurements considered for calibration. Most data are either taken for vertical incidence angles or corrected to vertical through model-dependent unfolding. Only publications with sufficient description of systematic uncertainties have been considered.

scale was introduced for BESS-TeV, CMS and DEIS, since none was reported in their publications, with a presumed error of $5 \%$.

A test was performed on each set to decide whether they are compatible with other data sets and their reported errors are properly estimated. The vertical fluxes and charged ratios, with zenith angles between $0-20^{\circ}$, were combined as a single data set, and their average flux and charge ratio were fit with a cubic spline distributing the knots evenly in $\log _{10}(E / \mathrm{GeV})$. The $\chi^{2}$ of the sets with respect to these two average expectations were minimized varying the corrections on the experiment's systematic parameters. From this exercise we find that the combined fit of vertical fluxes, including AMS-02, BESS-TeV and L3+cosmic, returned a negligible small $p$-value. By varying the experiment combinations, we learned that AMS-02 was responsible for the disagreement and excluding it results in a $p$-value of over $90 \%$. The vertical muon charge ratio, on the other hand, had a $p$-value of $60 \%$ when all experiments were included, increasing to $90 \%$ when AMS-02 was removed. From this test we conclude that all data sets are indeed compatible with each other with the exception of AMS-02, so these data were excluded from the final calibration.

\section{Calibrating neutrino fluxes with muons}

We use the cascade code MCEQ $[13,14]^{1}$ to perform the calculations of muon and neutrino fluxes. The calibration is done by fitting the muon fluxes produced by MCEQ with those in the data by varying a set of parameters that govern the production of these fluxes as well as parameters that correct the experimental data. The test statistic is a modified $\chi^{2}$ given by

$$
\chi^{2}=\sum_{i}^{N} \frac{\left(O_{i}-E_{i}\right)^{2}}{\sigma_{O_{i}}^{2}}+\sum_{j}^{M} \frac{\left(F_{j}-E_{j}\right)^{2}}{\sigma_{F_{j}}^{2}}
$$

where the first sum compares observation $O_{i}$ at each data point $i$ with expectation $E_{i}$ from MCEQ divided by the error in the observation $\sigma_{O_{i}}$. The second sum penalizes deviations of the parameter $F_{j}$ from their expectation $E_{j}$ divided by its estimated error $\sigma_{F_{j}}$.

The MCEQ fluxes are produced for each experiment using their reported zenith angles, location, altitude, and atmospheric conditions (averaging over the duration of data-taking) using NRLMSISE00 [15]. The cosmic ray primaries are modeled using the Global Spline Fit (GSF) [16]. GSF is

\footnotetext{
${ }^{1}$ https://github.com/afedynitch/MCEq
} 
a data-driven approach that compiles direct and indirect measurements of cosmic ray primaries to produce the flux of nucleons at the top of the atmosphere. The approach to obtain the model is similar to the present work since different cosmic ray experiments are cross-calibrated through a combined fit. For hadronic interactions, we use the new Data Driven Model (DDM), which is a fit of accelerator data of proton-carbon collisions taken by NA49 and NA61 experiments at CERN (for more details, see a different contribution at this conference [17]).

A novel feature of DDM is the parameterization of hadronic errors directly from data, which it exposes as ten parameters. These parameters modify the production of hadrons within atmospheric cascades imposing changes to the fluxes of muons and therefore also that of neutrinos. Each parameter modifies particle yields away from their central value (best fit of the NA49/61 data) to the edges of the reported $1 \sigma$ error bands. One additional parameter changes the total nucleon flux predicted by GSF within its error band. As a spline-based approach, GSF has many parameters. Our choice to only vary the total nucleon flux neglects the related uncertainty to the neutron fraction, which is a shortcoming of the current approach and will be handled in a later work. The current parameterization corresponds, in principle, to an energy-dependent spectral index variation, as done in $[1,3]$.

The yields of charged $\pi^{ \pm}$and $K^{ \pm}$are modified independently for each particle type, with a total of 8 parameters for the low energy part, corresponding to NA61 data taken at $31 \mathrm{GeV} \mathrm{[18],}$ and a high energy part corresponding to NA49 data at $158 \mathrm{GeV}[19,20]$, respectively. Two additional parameters modify the production of protons and neutrons in the cosmic showers within the uncertainties of the NA49 $158 \mathrm{GeV}$ data. The procedure used to compute the effects of these parameters on the data has been reported here [21].

A total of 25 parameters were used in the fit, where 16 of them were corrections to the data that account for reported systematic uncertainties. The fit was done using iMinuit [22], and the goodness-of-fit of the results was estimated using only the first term of Eq. (1).

\section{Results on muon and neutrino fluxes}

The fit was conducted simultaneously on all the experiments listed in Table 1, minus AMS-02. The values obtained result in an excellent fit, with a $p$-value for the data to originate from the model of $72 \%$ ( $\left.\chi^{2}=398 / 416 \mathrm{dof}\right)$. The result of the fit to the muon data as well as the starting point of the fit and data are shown in Figs. 1 and 2. The uncalibrated DDM+GSF consistently underestimates the fluxes, particularly at vertical directions, and it overestimates the muon charge ratio. The calibrated model, on the other hand, can explain the muon fluxes from vertical and horizontal directions simultaneously, as well as the charge ratio data.

The parameters governing corrections on the experimental data are mostly within two standard deviations, except for two parameters coming from L3+cosmic. This can be understood from Fig. 1, which shows that the data from $\mathrm{L} 3+$ cosmic requires corrections that significantly decrease its flux around $100 \mathrm{GeV}$. From the parameters responsible for the muon flux, and simultaneously the neutrino flux, those that modify the production of neutrons, high energy $\pi^{-}$and high energy $K^{+}$are pulled to produce higher yields by about $4 \sigma$. We think this can be partially explained by the fact that the parameterization of GSF errors at this moment is too simple, e.g. neglecting a neutron fraction uncertainty to account for the error in proton to helium ratio in cosmic rays, and discrepancies there 
are captured by the remaining fit parameters. The rest of the parameters of interest are fit within 2 standard deviations from their expectation.

The resulting vertical down-going neutrino fluxes are shown in Fig. 3 together with the uncalibrated GSF+DDM output as well as the flux from two commonly used calculations, the HKKMS model [23] and the Bartol model [24]. The calibrated flux is close to the HKKMS flux for $v_{e}$ at all energies. For $v_{\mu}$, the fluxes agree below $2 \mathrm{GeV}$, and for energies above this, the calibrated flux is lower. In both cases, the calibrated output produces a higher flux than the original GSF+DDM.

Error bands accompany both the calibrated and original fluxes, although the errors for the calibrated model are too small to be appreciated in the figure. Below $100 \mathrm{GeV}$, the main source of error are the yields of low energy $\pi^{+}$and $\mathrm{K}^{+}$, introducing an uncertainty between $1 \%$ and $4 \%$ on the neutrino fluxes. Above $100 \mathrm{GeV}$ the error on the cosmic ray primary flux from GSF becomes dominant, resulting in a $6 \%$ uncertainty at $100 \mathrm{TeV}$. For comparison, prior to the calibration, the error on GSF+DDM was about $10 \%$ at $1 \mathrm{TeV}$.

The ratio $v_{\mu} / \bar{v}_{\mu}$ for vertical down-going neutrino fluxes is shown in Fig. 4 in a similar fashion as the previous figures. The calibrated GSF+DDM calculation predicts a ratio that is significantly higher than HKKMS, although it is still lower than the Bartol results. The errors in this ratio are also reduced by the muon data.
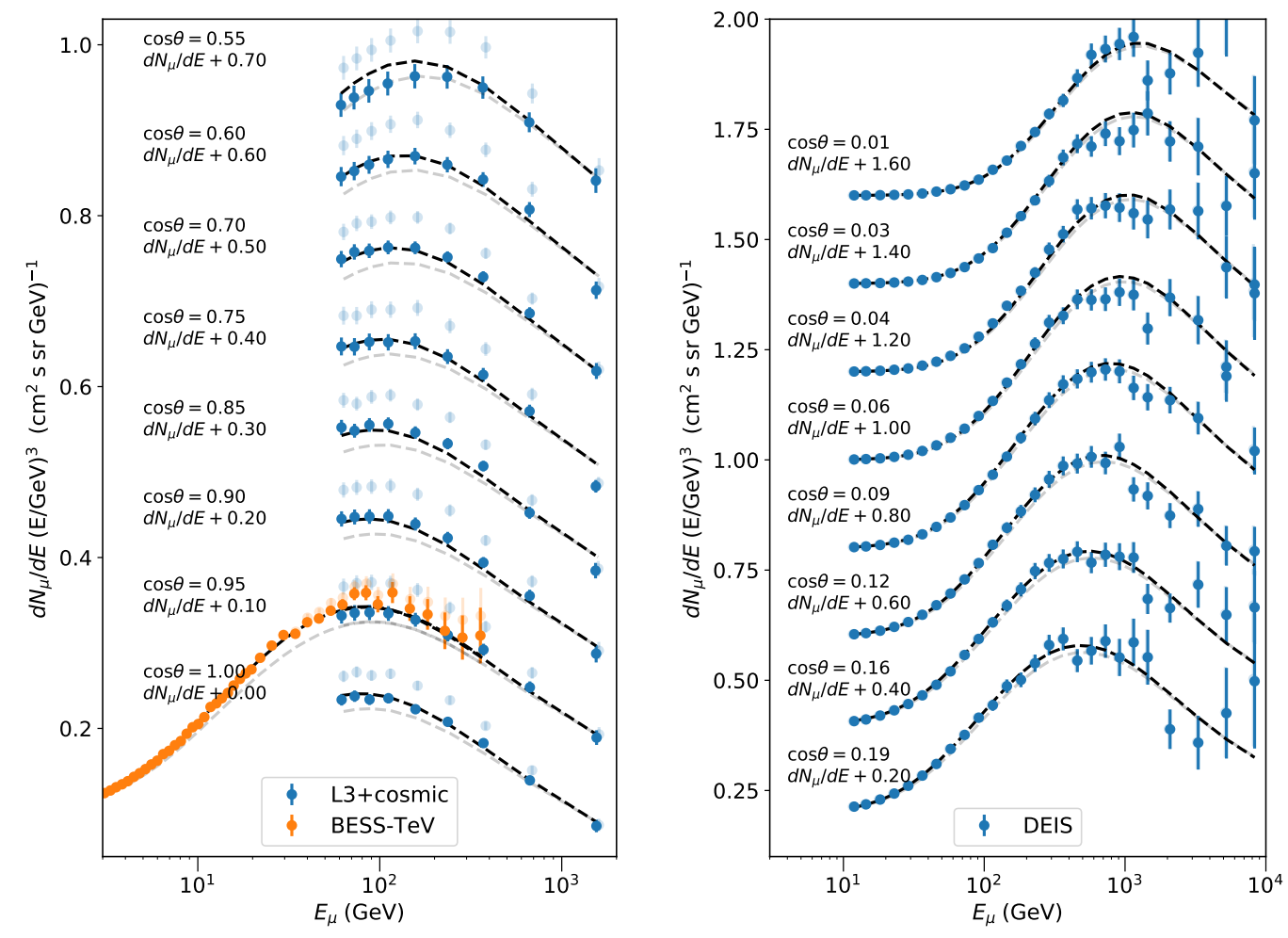

Figure 1: Muon fluxes for near vertical (left) and horizontal (right) incoming directions. Solid colors correspond to the experimental data and the flux calculation after the fit. The data and fluxes prior to the fit are shown with a transparency. A factor has been added to fluxes from different incoming directions to be able to show them in the same figures. 


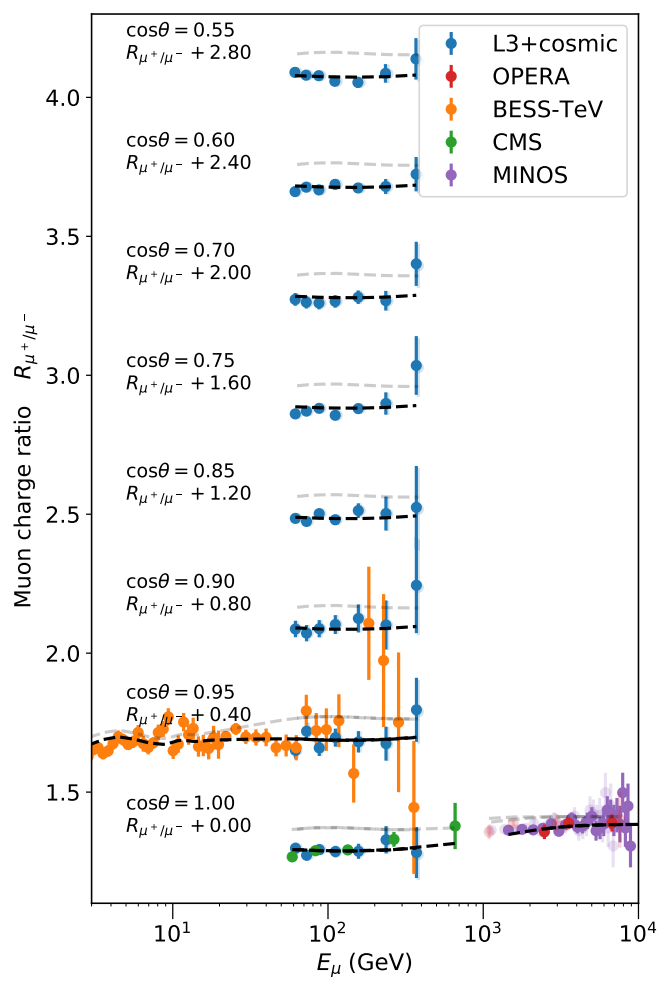

Figure 2: Muon charge ratio for near vertical incoming directions. Solid colors correspond to the experimental data and the charge ratio calculation after the fit. The data and ratios prior to the fit are shown with a transparency. A factor has been added to fluxes from different incoming directions to be able to show them in the same figure.
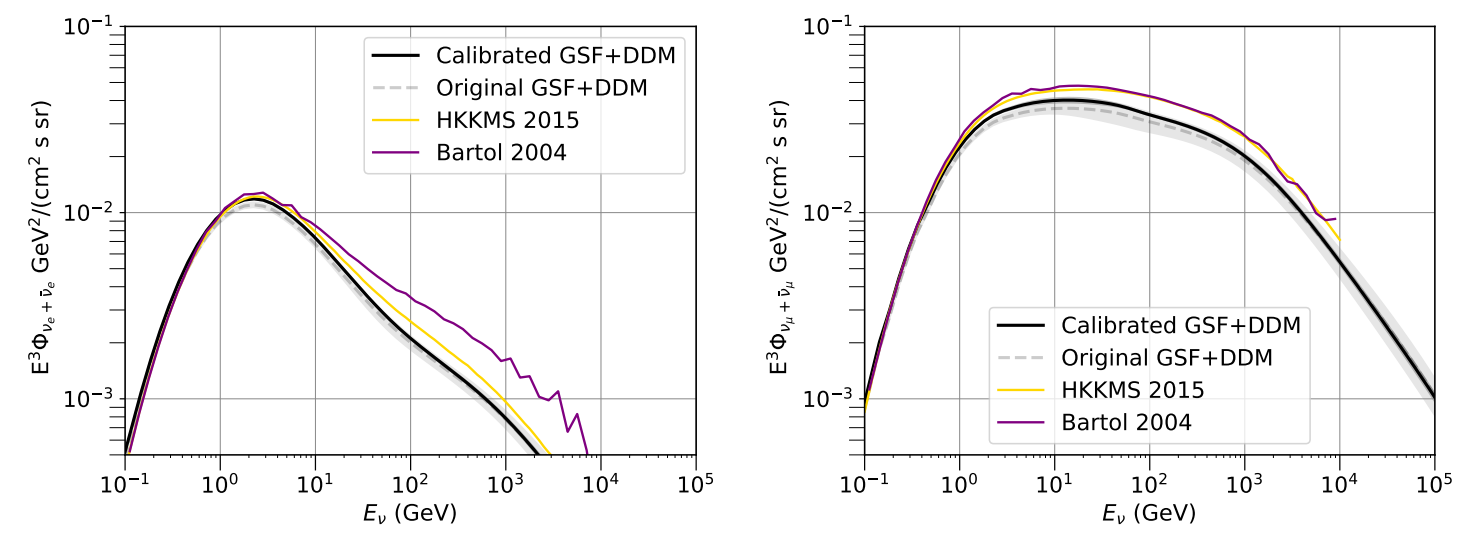

Figure 3: Vertical down-going fluxes of electron (left) and muon (right) neutrinos resulting from the calibration of the GSF+DDM output. The original output of GSF+DDM is shown in gray. The shaded regions depict the errors of the calculation. Fluxes from HKKMS and Bartol computations are shown for comparison. 


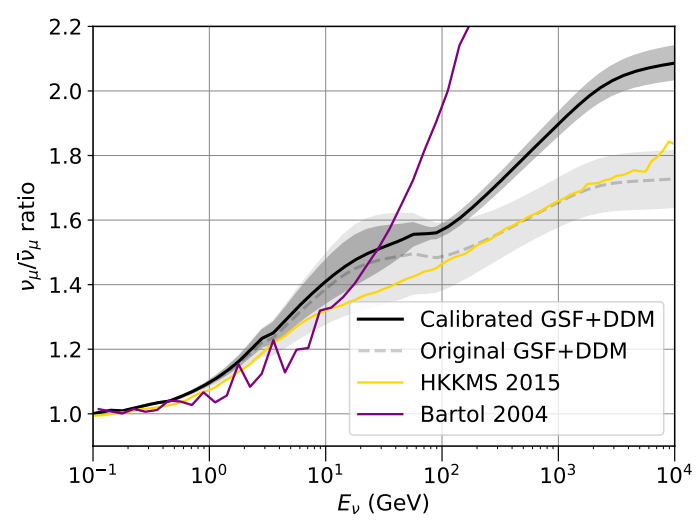

Figure 4: Ratio $v_{\mu} / \bar{v}_{\mu}$ for vertically down-going neutrinos as function of energy. The output from both original and calibrated GSF+DDM calculations are shown. The shaded regions depict the errors of the calculation. Expected ratio from HKKMS and Bartol computations are shown for comparison.

\section{Discussion and future work}

We have successfully used muon spectrometer data to calibrate an atmospheric neutrino flux while simultaneously explaining the muon flux and muon charge ratio $\mu^{+} / \mu^{-}$at both vertical and horizontal incoming directions. The calibration was done adjusting the primary cosmic rays, yields of light mesons and experimental parameters that modify the interpretation of the data. Some of our best fit values suggest modifications that are stronger than expected, but we think this is mainly due to the simplicity in the parameterization of the errors coming from the cosmic ray flux. Using these corrections, we compute a calibrated neutrino flux with errors below $6 \%$ at all energies.

To further verify the resulting fluxes and their small error, future work will introduce a more sophisticated handling of the errors reported in GSF as well as the inclusion of additional data from near horizontal fluxes, since this region is currently constrained by a single experiment. Apart from that, the inclusion of muon flux measurements from deep underground detectors will also be explored.

\section{References}

[1] J. Evans, D. G. Gamez, S. D. Porzio, S. Söldner-Rembold, and S. Wren, Phys. Rev. D 95 (2017) 023012.

[2] T. K. Gaisser and M. Honda, Ann. Rev. Nucl. Part. Sci. 52 (2002) 153-199.

[3] G. D. Barr, T. K. Gaisser, S. Robbins, and T. Stanev, Phys. Rev. D74 (2006) 094009.

[4] M. Honda, T. Kajita, K. Kasahara, S. Midorikawa, and T. Sanuki, Phys. Rev. D75 (2007) 043006.

[5] M. Duranti. PhD thesis, INFN, Perugia, 2012.

[6] S. Haino et al., Phys. Lett. B 594 (2004) 35-46. 
[7] CMS Collaboration, V. Khachatryan et al., Phys. Lett. B692 (2010) 83-104.

[8] L3 Collaboration, P. Achard et al., Phys. Lett. B598 (2004) 15-32.

[9] O. C. Allkofer, H. Jokisch, G. Klemke, Y. Oren, R. Uhr, G. Bella, and W. D. Dau, Nucl. Phys. B259 (1985) 1-18. [Erratum: Nucl. Phys.B268,747(1986)].

[10] MINOS Collaboration, P. Adamson et al., Phys. Rev. D76 (2007) 052003.

[11] OPERA Collaboration, N. Agafonova et al., Eur. Phys. J. C74 (2014) 2933.

[12] M. Unger. Phd thesis, 2, 2004.

[13] A. Fedynitch, R. Engel, T. K. Gaisser, F. Riehn, and T. Stanev, PoS(ICRC2015)1129 (2015).

[14] A. Fedynitch, F. Riehn, R. Engel, T. K. Gaisser, and T. Stanev, Phys. Rev. D 100 (2019) 103018.

[15] J. M. Picone, A. E. Hedin, D. P. Drob, and A. C. Aikin, Journal of Geophysical Research: Space Physics (1978-2012) 107 (Dec., 2002) SIA 15-1-SIA 15-16.

[16] H. P. Dembinski, R. Engel, A. Fedynitch, T. Gaisser, F. Riehn, and T. Stanev, PoS(ICRC2017)533 (2018). [35,533(2017)].

[17] A. Fedynitch and M. Huber, PoS(ICRC2021)1076 (2021).

[18] NA61/SHINE Collaboration, N. Abgrall et al., Eur. Phys. J. C 76 (2016) 84.

[19] NA49 Collaboration, C. Alt et al., Eur. Phys. J. C49 (2007) 897-917.

[20] NA49 Collaboration, T. Anticic et al., Eur. Phys. J. C 68 (2010) 1-73.

[21] J.-P. Yáñez, A. Fedynitch, and T. Montgomery, PoS(ICRC2019)881 (2020).

[22] iminuit team, "iminuit - a python interface to minuit." https://github.com/scikit-hep/iminuit. Accessed: 2018-03-05.

[23] M. Honda, M. S. Athar, T. Kajita, K. Kasahara, and S. Midorikawa, Phys. Rev. D92 (2015) 023004.

[24] G. D. Barr, T. K. Gaisser, P. Lipari, S. Robbins, and T. Stanev, Phys. Rev. D 70 (2004) 023006. 